\title{
Embolisation of a complex coronary arteriovenous fistula in a 6 year old child: the need for specialised embolisation techniques
}

\author{
J F Reidy, M J Tynan, S Qureshi
}

\begin{abstract}
Transcatheter embolisation was performed in a six year old child with a large haemodynamically significant arteriovenous fistula between the left coronary artery and right ventricle. The fistula seemed to fill via a single latge septal branch of the left anterior descending coronary artery. This was occluded distally with a detachable balloon backed up by a proximal coil. After this the flow through the fistula was considerably reduced but it still filled via a small diagonal branch that had not been noted previously. This branch was subsequently occluded in its most distal part by a coaxial catheter technique and a single microcoil. Complete occlusion of the fistula was produced and there were no complications.
\end{abstract}

Despite the recent development of interventional procedures in congenital heart disease and the increasing use of embolisation techniques there are few descriptions of embolisation of coronary arteriovenous fistulas and malformations. ${ }^{12}$ Most reviews of interventional procedures in congenital heart disease make no mention of embolisation of coronary artery anomalies. ${ }^{34}$ We have previously described cases in which embolisation by a detachable balloon was used to occlude large coronary arteriovenous fistulas. ${ }^{12}$ We describe a further unusual case in a six year old child who had a complex arteriovenous fistula with a dual supply that needed two different embolisation techniques to achieve complete occlusion.

Department of Radiology, Guy's Hospital, London J F Reidy

Department of Paediatric Cardiology, Guy's Hospital, London M J Tynan $S$ Qureshi

Correspondence to DrJ F Reidy, Department of Radiology, Guy's Hospital, Guy's Hospital, London SE1 9RT.

A six year old Nigerian boy was found to have a murmur when he was a year old, when he was examined after a chest infection. The murmur was continuous with accentuation in diastole and was best heard at the lower left sternal border. Otherwise he was symptom free, active, and thriving.

Cardiac catheterisation was performed when he was three years old. Pulmonary artery pressure was $38 / 12$ (mean 24), right (mean 65) $\mathrm{mmHg}$. Oximetry showed a left to right shunt (Qp:Qs 2·5:1). Aortography showed a fistula between the left anterior descending coronary artery and the apex of the right ventricle.

When he was six years old his resting electrocardiogram and chest radiograph were normal. A first pass radionuclide scan showed the left to right shunt $(2 \cdot 6: 1)$.
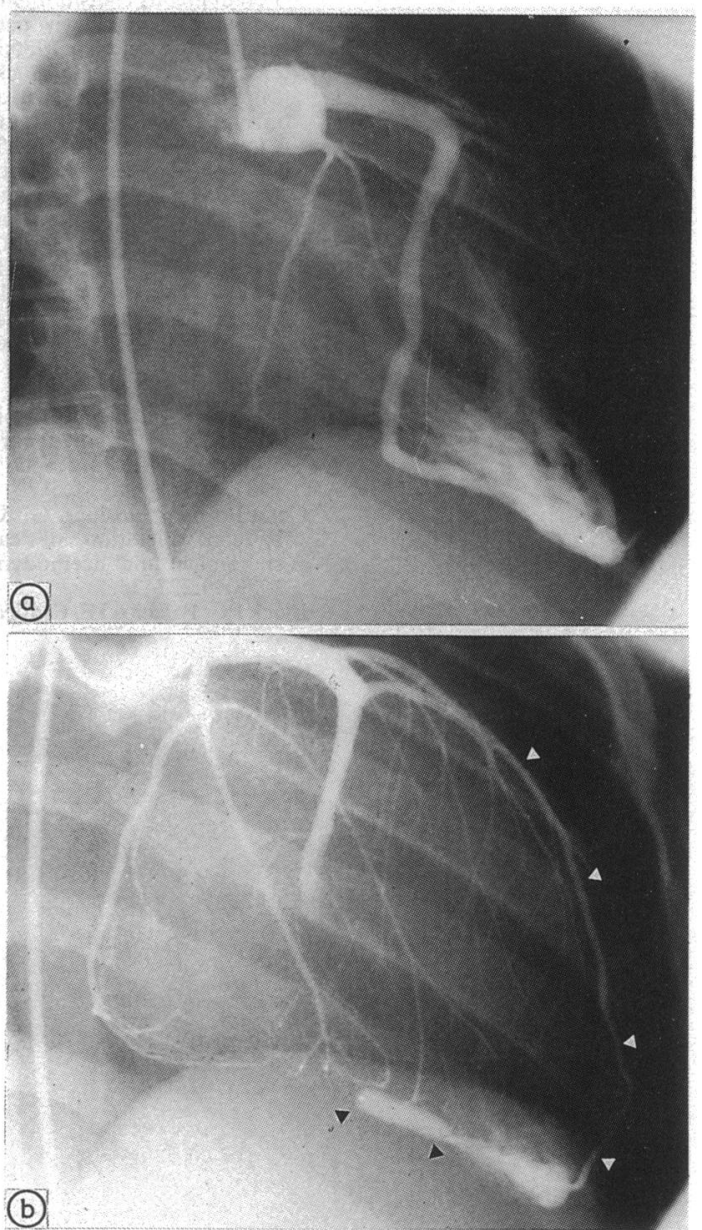
ventricular pressure 40/10, and aortic $90 / 50$
Figure 1(a) Selective left coronary arteriogram (right anterior oblique view) that shows a single large septal branch arising from the proximal anterior descending artery and communicating with the right ventricle. Note the poor filling of the other branches of the left coronary caused by the steal effect. (b) $A$ contrast filled detachable balloon (black arrowheads) has occluded the fistulous artery in a distal location. Despite this and considerably reduced flow the fistula is still filled by a much smaller diagonal branch (white arrowheads). This is now shown much more clearly that in $(1 a)$. 


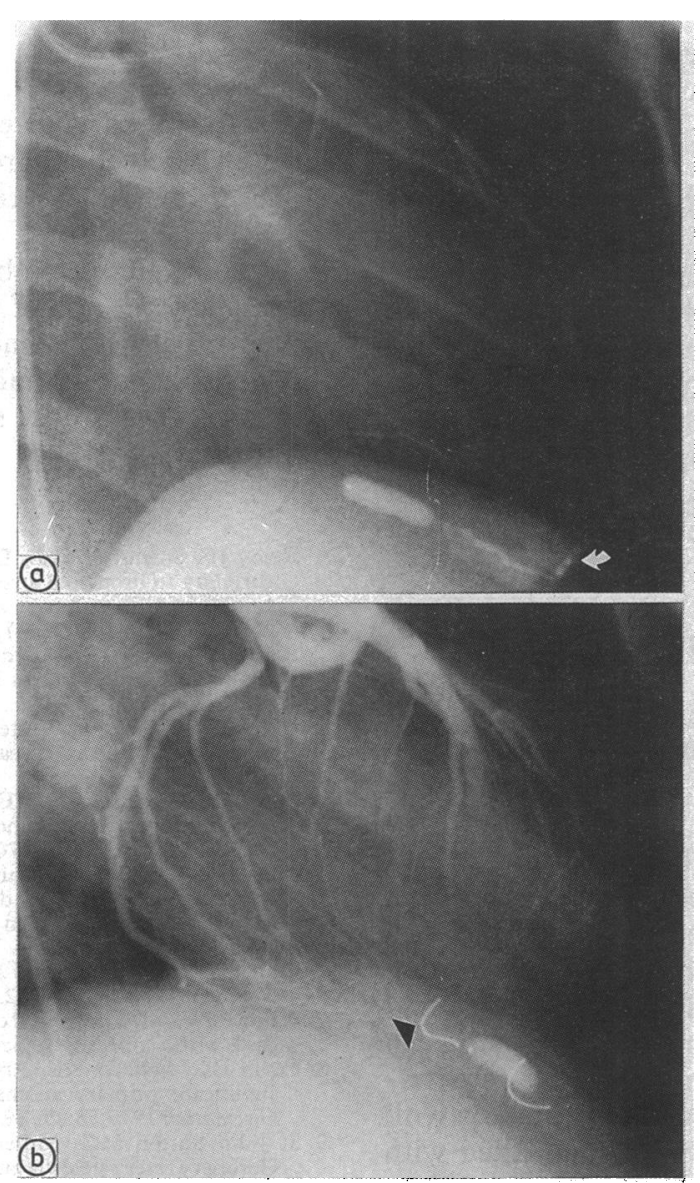

Figure 2 (a) A coaxial $3 F$ catheter has been passed into the distal part of the small diagonal branch. A metal marker identifies its tip (white arrow). The limited injection of contrast that is possible through this very small catheter opacifies the fistula. (b) Post embolisation. $A$ further microcoil has been placed immediately proximal to the balloon (arrow). The second coil is at the apex of the heart adjacent to the fistula. Note the much better filling of the normal coronary branches after occlusion of the fistula.

\section{EMBOLISATION PROCEDURE}

A $6 \mathrm{~F}$ catheter was positioned in the proximal left coronary artery via the femoral artery and under general anaesthesia. Coronary arteriography showed a large septal branch passing to the posterior interventricular groove and then forwards to drain into the right ventricle (fig la). Through this we guided the $2 / 3 \mathrm{~F}$ balloon catheter delivery system with an attached detachable latex balloon (Ingenor, Paris) into the large artery to a position just proximal to the fistula. A test inflation of the balloon showed that it produced complete occlusion and there were no untoward clinical effects or electrocardiographic abnormalities. The balloon, filled with dilute contrast medium, was then detached. A post embolisation cineangiogram showed occlusion of the large septal artery but some residual filling of the fistula via a much smaller and previously unrecognised diagonal branch arising from the anterior descending artery (fig 1b). We used a 3 French coaxial catheter (Tracker 18) (Target Therapeutics) and a steerable 0.014 inch coronary guide wire to catheterise this branch selectively so that the tip of the $3 F$ catheter was immediately proximal to the site of the fistula (fig 2a). A single platinum coil (Hilal $1.5 \mathrm{~cm}$ length)
(William Cook, Europe) was placed here. Because the small detachable balloon was inflated to its maximum capacity with contrast medium and not with liquid silicone monomers we decided to position a further microcoil immediately proximal to the detachable balloon. This was to prevent any possible recanalisation in the event of early deflation of the balloon. After this no filling of the fistula was seen (fig $2 \mathrm{~b}$ ). The left coronary artery and its branches were now seen much more clearly than before the embolisation procedure when there had been a "steal effect".

Immediately after the embolisation there was transient junctional rhythm, but no other ST segment changes. Isosorbide dinitrate $(250 \mu \mathrm{g})$ was injected into the left coronary artery.

After the procedure, the serial electrocardiograms showed transient $T$ wave inversion, which reverted to normal three days later. Serum creatine kinase peaked (325 U/1 (normal $<205 \mathrm{U} / 1)$ ) 24 hours after the procedure and then returned to normal. A technetium pyrophosphate scan was normal two days after the procedure. There were no complications and the child made an uneventful recovery. A chest radiograph one day later showed that the balloon had deflated but was still in position. The murmur was not heard and no flow through the malformation was detected on Doppler echocardiography. At review two months later the patient was well and no murmur was heard. A chest radiograph showed the coils and balloon still in the same position.

\section{Discussion}

Primary congenital coronary fistulas to cardiac chambers or great vessels are rare. They are found in about one in every 500 patients having coronary arteriography. ${ }^{5-7}$ They are the most common haemodynamically significant congenital coronary anomaly; in one series 21 fistulas were found in 34 cases. ${ }^{8}$ Half of 363 coronary arteriovenous fistulas arose from the right coronary artery and $42 \%$ arose from the left coronary artery. Drainage was to any cardiac chamber but most commonly it was to the right ventricle $(41 \%) .8$

As in our patient the condition most commonly presents as a murmur in a symptom free child. ${ }^{7}$ Because complications in adult life can cause premature death the condition is usually treated surgically. Cardiac failure is the most common complication and increases with age. ${ }^{7}$ Coronary ischaemia and angina can occur because of the steal effect of the fistula. ${ }^{9}$ Though the resting electrocardiogram was normal in our patient, coronary arteriography showed the branches of the left coronary artery much better after the embolisation. Infective endocarditis ${ }^{710}$ and atrial fibrillation can occur $^{10}$ and even rupture has been described. ${ }^{11}$ As a result of these risks early prophylactic operation has been advocated. ${ }^{7}$ Mortality from these operations in low, especially when the fistulas are on the inferior surface of 
the heart; even so the operation requires cardiopulmonary bypass with its consequent morbidity.

There are two specific technical requirements for embolisation of a coronary arteriovenous fistula. Firstly, the point of occlusion must be distal in the feeding artery and as close to the fistula as possible so that the risk of occluding normal coronary branches is low. These may not be so obvious due to the steal effect of a large fistula. Secondly, occlusion of a large high flow artery at a specific point is needed; this can be difficult and a detachable balloon is the ideal method. This allows immediate occlusion to be effected and is reversible if it is not satisfactory. In addition, it does not require more dangerous selective catheterisation of distal coronary branches with larger catheters.

The initial study in our patient showed that the coronary fistula was supplied by a single large septal branch. But an injection of contrast medium after embolisation also showed a much smaller second feeding branch. Had an operation been performed this second branch might easily have been missed. Embolisation methods have improved in recent years so that now even a small coronary artery branch such as this in a child can be selectively catheterised and occluded at the site of the fistula with a single microcoil. A latex balloon filled with contrast medium rather than silicone polymers was needed to occlude the large feeding branch. To prevent early deflation and recurrence of the fistula we placed a further coil proximal to the balloon. This coil alone would almost certainly not have produced occlusion.

When coronary arteriovenous fistulas are haemodynamically significant and a decision has been made to treat then embolisation should be considered the treatment of first choice. Modern embolisation techniques, which allow the use of more than one type of occlusive device, enable a precise intraoperative diagnosis and successful occlusion of fistulas-even those supplied by more than one artery.

1 Reidy JF, Sowton E, Ross DN. Transcatheter occlusion of coronary to bronchial anastomosis by detachable balloon combined with coronary angioplasty at same procedure. Br Heart J 1983;49:284-7.

2 Reidy JF, Jones ODH, Tynan MJ, Baker EJ, Joseph MC Embolisation procedures in congenital heart disease. $B r$ Heart J 1985;54:184-92.

3 Mitchell SE, Kan JS, White RI. Interventional techniques in congenital heart disease. Semin Roentgenol 1985;3: in congenit

4 Perry SB, Keane JF, Lock JE. Interventional catheterisation in pediatric congenital and acquired heart disease. $\mathrm{Am} \mathrm{J}$ Cardiol 1988;61:109G-17G.

5 Effler DB, Sheldon WC, Turner JJ, Groves LK. Coronary arteriovenous fistulas: diagnosis and surgical management. Report of fifteen cases. Surgery 1967;61:41-50.

6 Baltaxe HA, Wixson D. The incidence of congenital anomalies of the coronary arteries in the adult population. Radiology 1977;122:47-52.

7 Wilde P, Watt I. Congenital coronary artery fistulae: six new cases with a collective review. Clin Radiol 1980;31:301-11.

8 Levin DC, Fellows KE, Abrams HL. Haemodynamically significant primary anomalies of the coronary arteries. Circulation 1978;58:25-34.

9 St John Sutton MG, Miller GAH, Kerr IH, Traill TA. Coronary artery steal via large coronary artery to bronchial artery anastomosis successfully treated by operation. $\mathrm{Br}$ artery anastomosis succes

10 McNamara JJ, Gross RE. Congenital coronary artery fistula. Surgery 1969;65:59-69.

11 Haberman JH, Howard ML, Johnson ES. Rupture of the coronary sinus with haemopericardium. Circulation 1963;28:1143-4. 